\title{
Scolia
}

Revue de linguistique

$32 \mid 2018$

Autour des pseudos-clivées

\section{L'emploi de ce dans les reprises de contenu propositionnel}

\section{Claude Muller}

\section{(2) OpenEdition}

1 Journals

\section{Édition électronique}

URL : http://journals.openedition.org/scolia/337

DOI : $10.4000 /$ scolia.337

ISSN : 2677-4224

Éditeur

Presses universitaires de Strasbourg

\section{Édition imprimée}

Date de publication : 3 juillet 2018

Pagination : 117-138

ISBN : 979-10-344-0021-8

ISSN : $1253-9708$

\section{Référence électronique}

Claude Muller, «L'emploi de ce dans les reprises de contenu propositionnel », Scolia [En ligne], 32 I

2018, mis en ligne le 09 avril 2019, consulté le 10 décembre 2020. URL : http://

journals.openedition.org/scolia/337 ; DOI : https://doi.org/10.4000/scolia.337

\section{(c) (i) (3)(2)}

Les contenus de la revue Scolia sont mis à disposition selon les termes de la Licence Creative Commons Attribution - Pas d'Utilisation Commerciale - Partage dans les Mêmes Conditions 4.0 International. 
SCOLIA $32 / 2018$, p. 117-138.

\title{
L'emploi de ce dans les reprises de contenu propositionnel
}

\author{
Claude MULler \\ Université Bordeaux Montaigne, UMR 5263 CLLE \\ claude.muller31@orange.fr
}

\section{Introduction}

La reprise d'un contenu propositionnel établit une relation anaphorique particulière, puisque son point de départ n'est pas une expression de même statut catégoriel que le support anaphorique: il s'agit de passer d'une expression phrastique, souvent de délimitation incertaine, à sa représentation sous forme nominalisée, ce qui s'accompagne d'opérations mentales de prise en compte du contenu global de la proposition. La reprise anaphorique sous forme pronominale s'accompagne aussi de modifications dans le contenu de l'information: la structure en topique et commentaire, ou en thème et rhème du contenu propositionnel, disparaît obligatoirement. En principe, cette modification est associée au dynamisme communicatif, qui insère l'anaphore dans un nouveau schème prédicatif. C'est là que ce présente des particularités intéressantes: sauf utilisation comme sujet du verbe être, et parce qu'il est toujours d'emploi contraint, il n'est pas utilisable librement en construction verbale. Cette situation n'est pas une limite à son utilisation: élément libre dans diverses constructions, ou introducteur de subordonnées relatives particulières du type des relatives «libres», il est devenu un outil majeur de construction de schèmes syntaxiques divers associant à un noyau prédicatif initial des 
développements très variés. On examinera d'abord les constructions, ensuite on essaiera d'en donner des éléments d'analyse.

\section{Les constructions}

Ce sont les formes non clitiques cela, ça qui sont les formes libres de reprise de contenu propositionnel dans les constructions verbales. Le clitique sujet ce est une variante réservée au verbe être (\$2.1.1.). Il existe aussi des emplois cataphoriques, notamment avec des complétives, on les laissera de côté ici. Les constructions caractéristiques des emplois de ce qui nous intéresseront sont les constructions en phrase non verbale (\$1.2.), dans des relatives «libres» (\$1.3.), dans un type de clivée (\$1.4.), dans quelques constructions à participe présent (\$1.5.), avec sur (\$1.6.), avec une construction figée à l'infinitif (\$1.7.). Cette partie donnera des exemples caractéristiques, les constructions étant examinées dans la troisième partie. Le contenu propositionnel référent est mis en valeur typographiquement dans les exemples de cette partie, cette distinction n'étant pas toujours évidente ni certaine comme on le verra.

\subsection{La reprise libre de contenu propositionnel}

Cette reprise ne peut employer ce, mais ceci ou cela:

1) Salagnon continua de dessiner; cela se sut et on lui demanda des portraits. (Jenni, L'art français de la guerre, 2011) ${ }^{1}$

ou encore ça plus fréquent à l'oral (Guillot, 2006: 65):

2) j'ai pris la rue qu'i m'a montrée (...) ça nous f'sait gagner $d u$ temps hein

Le pronom ce est employé de façon limitée en tant que clitique sujet, surtout avec être:

3) Sancey fut pris. Ce fut facile. (Jenni)

\subsection{Reprise de contenu propositionnel par ce devant un circonstanciel}

L'exemple suivant de Berrendonner (1990: 35) illustre le rôle de ce:

1 Les exemples à auteur et date sont tirés de Frantext. Lorsque la même œuvre est citée on pourra ne noter que le nom de l'auteur. 
L'EMPLOI DE CE DANS LES REPRISES DE CONTENU PROPOSITIONNEL

4) Anna a sept ans durant les années 1960, ce dans un bled au pied des Dents-du-Midi.

Le circonstanciel de lieu n'est pas directement attaché à la phrase verbale: le ce représente cette dernière sous forme nominalisée. Son statut en tant que classe de mots n'est pas clair: il semble relativement indépendant de ce qui suit mais il faut une suite, ce qui fait penser à un «clitique syntagmatique». Le lien avec la suite est une construction averbale où le prédicat est implicite: "a eu lieu», "s'est passé». La construction disloquée forme une seule phrase énonciative, où l'assertion initiale, autonome, est suivie d'un segment juxtaposé non autonome (mais la coordination aurait été possible).

Quel statut pour ce segment? Sans ce, il s'agirait d'un banal circonstanciel détaché. Ici, on a un thème à contenu propositionnel et le complément circonstanciel d'un prédicat absent. On peut hésiter sur la structure: simple absence de prédicat pour former une petite phrase, ou structure nominale dont la tête serait $c e$ ?

4a) [...] (et) cela (se passe) dans un bled.../ (chose/évènement qui se passe) dans un bled [...].

On ne pourrait pas tellement dans le second cas utiliser ce:

4b) ??Anna a sept ans durant les années 1960, ce qui se passe dans un bled au pied des Dents-du-Midi.

Autres exemples:

5) [...] j’ai tous les documents secrets nécessaires qui montrent que je ravitaillais les bonnes personnes. Et ce, depuis assez longtemps pour que mon appartenance au bon côté ne puisse être mise en doute. (Jenni, L'art français de la guerre, 2011)

6) Dans le cas contraire, la mort survient obligatoirement et ce, dans un délai d'une vingtaine de minutes. (Simonet, Olivès, Beltra, Roman autour d'une disparition, 2013)

7) Neufheures, c'était déjà tard, même si on n'avait pas à se lever le lendemain pour traire les vaches, et ce pendant trois jours [...]. (Lafon, Les pays, 2012)

Ces exemples montrent le fonctionnement de ce relais: il rassemble le contenu nominalisé d'une partie de la clause. La construction présente fréquemment un et lorsque le démonstratif est suivi directement d'un circonstant. 


\subsection{Le ce figure devant une relative}

Il y a deux constructions. L'une comporte les formes que, qui, dont, l'autre des pronoms pleins et ce y est facultatif.

\subsubsection{Avec ce qui}

8) Au nôtre, le père P. (il s'agit du maître d'école), il manquait un bras, ce qui l'handicapait un peu pour distribuer des taloches. (Crocq, Une jeunesse en Haute-Bretagne, 2011)

9) (des réfugiés) Nous en abritons quelques-uns, ce qui n'est pas facile, car le pavillon n'est pas trop grand pour notre famille de sept personnes. (ibid.)

10) Notre mère avait mis à sa disposition la petite pièce mansardée du deuxième étage, dont la fenêtre donnait sur le jardin, ce qui la mettait à l'abri des regards et des indiscrétions. (ibid.)

La partie à quoi réfère ce est variable; parfois toute la proposition:

11) Les Allemands commencèrent à quitter la ville avant que l'aviation n'eût besoin d'intervenir, ce qui évita à Rennes de subir le sort des villes normandes. (ibid.)

parfois un morceau (une partie prédicative), comme ci-dessous:

12) Un professeur sortit, mais calmement et sans bondir hors de la classe, ce qui déjà m'étonna. (ibid.)

C'est le contenu de la relative qui délimite la partie anaphorisée; on comprend en (12) que ce qui étonne le narrateur, c'est le calme, pas la sortie du professeur.

13) Des avions alliés larguaient des bombes sur les convois allemands qui affluaient vers le front par les routes secondaires, et mitraillaient ce qui leur paraissait suspect, ce qui pouvait être le cas de nos baraquements. (ibid.)

14) Beaucoup de Rennais d'ailleurs n'aimaient pas non plus à l'époque entendre parler breton dans leur ville, ce qui pouvait être le cas entre des bretonnants qui s'y étaient installés [...]. (ibid.)

La référence peut se faire sur une hypothétique:

15) Si le beurre était épuisé, ce qui arrivait assez souvent, on faisait des tartines de saindoux pour la collation de quatre heures. (ibid.) 
L'EMPLOI DE CE DANS LES REPRISES DE CONTENU PROPOSITIONNEL

C'est possible aussi avec une interrogative indirecte (le ce peut donc référer aux deux propositions sous-jacentes, positive et négative comme en (16) ou à une seule comme en (17):

16) [...] le problème est maintenant de savoir si elle veut du café ou non, ce qui lui semble une question proprement blasphématoire au milieu du désastre. (Garat, Merle, 1996)

17) Valéry-Larbaud se demande si on observera le Jour de la Victoire jusqu'à la fin du siècle, ce qui lui paraît peu probable. (Grangaud, Calendrier des poètes, 2001)

\subsubsection{Avec ce que}

18) (nos maîtres) Ils n'étaient en général pas excessivement patients, ce que - avec le recul, je comprends parfaitement. (Crocq)

19) Quelques-uns recherchaient les nids et faisaient collection d'oufs, ce que mon copain et moi ne manquions pas de critiquer. (ibid.)

20) Si la solitude existe, ce que j'ignore, on aurait bien le droit, à l'occasion, d'en rêver comme d'un paradis. (Camus, dicocitations. com)

\subsubsection{Avec ce dont}

21) (mon père) Il éprouvait bien sûr notre patience, ce dont il ne tenait aucun compte, mais bientôt également celle de son interlocuteur. (Crocq)

\subsubsection{Avec des formes pleines de relatifs}

Il s'agit de quoi pour un contenu propositionnel:

22) [...] il devait passer quelques jours (ou instants, je ne sais plus exactement) en purgatoire pour quelques imperfections commises dans la récitation du bréviaire, ce sur quoi le Bon Dieu est très sévère pour les prêtres. (Artières, Vie et mort de Paul Gény, 2013)

Lefeuvre (2006) en donne des exemples, comme:

23) Certes il s'agit d'inciter les Canadiens à sortir de leur province natale et à découvrir leur propre pays dans un esprit d'unité nationale, ce à quoi ils sont, paraît-il, fort peu enclins. (Tournier, Les météores, cité par Lefeuvre, 2006 : 241) 
F. Lefeuvre remarque que dans d'autres constructions, les formes de type Prép+quoi ne permettent pas le ce:

24) Mais je dois rester encore un peu, sans quoi je leur ferais outrage. (Kessel, Le lion, dans Lefeuvre, 2006: 239)

25) Correction de copies jusqu'à 17h. Après quoi elle sortit. (Lefeuvre, 2006: 239)

26) Jugement de Michel Rocard sur François Hollande [...]: «Un pur produit de la culture mitterrandienne. Un cynisme où les idées sont sans importance, le seul problème étant de savoir naviguer entre elles pour trouver l'équilibre». Après quoi, l'ancien premier ministre a aussi donné son avis sur Martine Aubry» «Elle joue toujours perso. [...]». (ibid.: 239)

\subsection{Les structures de type clivée: $P, c^{\prime}$ est ce + relative}

\subsubsection{Avec ce qui}

27) La vie derrière soi, là que je suis. J'aborde la dernière phase. Combien d'années, nul ne le sait. Je sais pourtant qu'elles sont comptées. C'est ce qui compte. J'arrive, plus ou moins vite, au bout du rouleau. (Doubrovsky, Un homme de passage, 2011)

28) Mon problème est sanguin, étant sous anticoagulants, qu'il va falloir arrêter quelques jours pour que la plaie puisse se cicatriser. C'est ce qui m'angoisse un peu, gare aux caillots! (ibid.)

29) [...] la guerre ne m'impressionne plus; et quant à la mort, eh bien je me fous de la mort. Et Mariani aussi. C'est ce qui me donne cette indulgence pour lui. (Jenni)

30) Ils durent patienter encore un long moment avant de pouvoir rentrer en Angleterre. Mais, et c'est ce qui nous a permis de retrouver leur trace, ils s'étaient mis sous la protection de nos services, afin de pouvoir se réfugier à l'ambassade à tout moment... (Lévy, L'étrange voyage de Monsieur Daldry, 2011)

À noter, en (30), l'anticipation du ce sur le contenu propositionnel. La présence d'un premier terme paraît indispensable ici pour garder une construction liée. 
L'EMPLOI DE CE DANS LES REPRISES DE CONTENU PROPOSITIONNEL

\subsubsection{Avec ce que}

31) [...] ce trafic avec les campagnards était prohibé et considéré comme du marché noir. C'est ce que ma mère faisait régulièrement. (Crocq)

32) La prison m'avait changé. C'est ce qu'on murmurait dans mon dos... (Chalandon, Retour à Killybegs, 2011)

33) [...] Le consul vient ici? - C'est ce qu'il annonce dans son message. (Lévy)

\subsubsection{Avec ce à quoi}

34) J'étais bien embêté qu'il prenne mon conseil à la lettre. Mais je suis le narrateur: il faut bien que je narre. Même si ce n'est pas ce que je veux, même si ce n'est pas ce à quoi j'aspire [...]. (Jenni)

La clivée est ici accompagnée de la négation.

\subsubsection{Avec ce dont}

35) L'acte s'identifie donc avec l'énoncé de l'acte. Le signifié est l'identique du référent. C'est ce dont témoigne la clausule "par la présente». (Benveniste, La philosophie analytique et le langage, 1963)

36) - Que diriez-vous d'aller voir où en sont les travaux à l'église Notre-Dame? - Comment! s'écria le duc, ils ne sont pas encore terminés? - C'est ce dont nous nous rendrons compte. (Queneau, Les fleurs bleues, 1965)

\subsection{Ce devant participe présent}

Il y a deux constructions, l'une avec ce, l'autre plus marginale avec ce que. Le figement est grand, puisque peu de verbes sont utilisés, mais ce ne sont pas des verbes ordinaires, plutôt des verbes à sens général de verbe d'action ou de parole, et un verbe de perception:

\subsubsection{Ce faisant}

37) Alors on le voit tendre la main, dénouer un ruban entortillé et, ce faisant, converser avec l'enfant d'un air très naturel. (Garat, Chambre noire, 1990) 
38) Zola raconte la famille des Rougon-Macquart et, ce faisant, apprend la génétique de son temps. (Serres, Le tiers-instruit, 1991)

39) Alors, avec Max, on est allés lever le pouce à l'embranchement de la bretelle. Il s'appliquait bien, en faisant le geste. Ça lui creusait même une ride sur le front. Ce faisant, il m'expliqua ses théories sur le stop. (Giesbert, L'affreux, 1992)2

F. Lefeuvre a trouvé quelques occurrences de ce que faisant:

40) [...] et elle gagne beaucoup d'argent à laver la tête aux beaux seigneurs de la ville. Ce que faisant, elle entre en quelque manière dans la tradition du métier: non point que laver la tête des hommes, spécialité des femmes qui passaient pour légères, fût ordinairement l'affaire des couturières. (Faral, La vie quotidienne au temps de Saint Louis, Lefeuvre 2006: 251)

Elle cite aussi des emplois avec quoi, de tonalité archaïque:

41) Mais cet autre qui s'oppose à moi, je le range par commodité dans l'autre camp et ne prétends point agir sur lui sauf par la guerre. Ce en quoi faisant, tu durcis et forges ton adversaire. Et moi je dis qu'ami et ennemi sont morts de ta fabrication. (SaintExupéry, Citadelle, cité par Lefeuvre, 2006: 251)

\subsubsection{Ce disant}

L'expression renvoie à des paroles, discours direct comme (42) mais parfois aussi mental (43) ou indirect (44):

42) C'est le froid de l'Est qui vous attend. Et cela finira comme cela! Ce disant, il lui mit le poing sur la tempe, pour bien lui signifier qu'elle serait fusillée. (Auroy, Jours de guerre, 2008)

43) Bon, pensa-t-elle, posant des pièces sur la table, je vais les laisser papoter de boxe, de coups de poing directs du droit et du gauche, et rejoindre de ce pas mes pénates. Et ce disant in petto, elle se dirigeait droit vers les toilettes. (Garat, Dans la main du diable, 2006)

44) Avant qu'il ne raccroche, je lui dis que je l'aimais. Je n'en pensais pas un mot. Pas celui-là, ni de cette manière en tout cas. C'était pour lui faire plaisir. J'avais l'impression qu'il désirait que je

2 On a supposé que c'est l'action elle-même qui est le référent de ce faisant, pas les commentaires sur la manière de faire. 
L'EMPLOI DE CE DANS LES REPRISES DE CONTENU PROPOSITIONNEL

lui fasse une concession de cette sorte. Tout se déroulait entre nous comme si nous jouions la fin d'un film, peut-être parce que cela rendait opportunément irréelle la fin de sa vie. Mais ce disant, j'eus l'obscure sensation que je venais de prononcer sa condamnation et de hâter son départ. (Bouillier, Rapport sur moi, 2002)

\subsubsection{Ce que voyant}

L'expression vient logiquement après une description:

45) Puis la mère se déchaîne après D.D. [...], après moi qui héberge tout le monde et ne fous rien (ce qui est mon boulot), après toute la terre et les étoiles. Ce que voyant je file à la gare et y reste éternellement. (Fallet, Carnets de jeunesse, 1992)

46) Immédiatement, les habitants du village, armés des instruments qu'ils avaient sous la main, accoururent au secours de leurs rejetons. Ce que voyant, les Peuls se précipitèrent armés de leurs bâtons. (Bâ, Oui mon commandant!, 1994)

47) Les parents poussèrent le cheval dans la cour et firent avancer leur mulet, ce que voyant, les petites protestèrent. Pour faire plaisir à un passant brutal, fallait-il qu'un vieil ami comme le mulet fût obligé de quitter la ferme? (Aymé, Nouvelles complètes, 2002)

\subsection{Surce}

La référence à ce qui précède est très vague et très générale. L'expression agit comme un connecteur de successivité dans le temps, ou parfois comme une marque de rupture avec tout ce qui précède:

48) Vers 7 heures donc sur le clou du frater et vêtu en rude besogneux, vais éveiller Bébert. On descend à la cave et on perd un temps précieux à chercher l'amarre de la remorque. Sur ce, sa vieille déboule en hurlant comme une conne qu'elle nous en refuse l'usage. La salope!! (Fallet)

49) «Tu peux répéter? hurla-t-il. - J'ai le courage de mes opinions. Et je te le redis: j'l'admire. - Eh bien, hurla papa, t'iras l'admirer ailleurs». Sur ce, il gifla Fatima qui partit dans sa chambre en pleurnichant et en se tenant la joue. (Giesbert) 


\subsection{Pour ce faire}

L'expression est figée. Le contenu propositionnel est un but:

50) On avait vu des parachutistes, et cette dame devait aller prévenir la gendarmerie à $5 \mathrm{~km}$ de là et pour ce faire elle s'armait d'un fusil [...]. (Beauvoir, Journal de guerre, 1990)

51) Après quelques jours d'étouffante impuissance, je ressentis le besoin de l'appeler. Je me levais de ma chaise pour ce faire quand le téléphone sonna. C'était lui. (Duperey, Le voile noir, 1992)

\section{Eléments d'analyse}

\subsection{Les constructions sans mot $Q u$ -}

\subsubsection{Les prédications satellites averbales à topique ce}

On a signalé l'emploi de cela comme anaphore de contenu propositionnel, ce qui est simplement une des situations dans lesquelles on utilise ce démonstratif. Dans ces emplois, ce est assez strictement limité au seul verbe être (les emplois signalés par Grevisse $\$ 1153$ note 41 sont archaïques et sortis de l'usage courant). L'emploi de cela (ou de ceci) s'est généralisé, avec un renforcement qui n'a pas de signification particulière. Buridant (2000 : \$107) compare la construction de l'ancien français Ce ne di je mie avec celle qui est d'usage depuis le français classique: Je ne dis pas cela (Molière, Le Misanthrope, I, 2). On retrouve l'emploi antéposé de ce dans certaines des expressions vues plus haut, notamment les participes présents.

L'emploi du démonstratif, aussi bien cela que ce ou ça, est utilisable pour la reprise (ou l'annonce cataphorique) d'un contenu propositionnel, parce que ce contenu n'est pas nommé, et n'a pas de support signifiant. C'est l'explication proposée par Kleiber dans plusieurs textes, dont on retiendra Kleiber (1987: 118).

La construction non verbale peut maintenir ce parce que précisément le verbe est absent. Y a-t-il ellipse d'un verbe être sousentendu? On pourrait peut-être le supposer, parce que ce est la forme naturelle du démonstratif devant le verbe être. Il semble pourtant préférable de voir dans cette construction la poursuite de l'ancien emploi de la forme courte objet antéposée devant un participe présent formant un circonstanciel de temps: ce pendant, ce durant, avec un 
L'EMPLOI DE CE DANS LES REPRISES DE CONTENU PROPOSITIONNEL

appui de la forme courte sur la forme verbale, dans la syntaxe objetverbe de l'ancien français. Lorsque l'introducteur du complément temporel a perdu son sens verbal, la construction s'est maintenue et s'est généralisée à des circonstanciels divers. L'ensemble forme alors une clause averbale à emploi dépendant, associée à une première construction, qui forme le noyau énonciatif principal de l'ensemble.

La construction a été étudiée dans plusieurs travaux par HobækHaff. Je citerai ici celui de 2017 en référence. Se pose la question du coordonnant: le et est souvent pris comme obligatoire. Il me semble qu'il illustre le statut fréquent de coordonnée de la clause non verbale, mais cette coordination peut éventuellement ne pas être marquée (comme dans l'exemple (4) cité de Berrendonner 1990). Dans son étude, Hobaek-Haff conteste aussi que le et soit obligatoire. Elle a trouvé un cas avec mais:

52) Il finira par accepter, mais ce dans le but de protéger la femme qu'il aime. (Hobæk-Haff, 2017: 92)

et signale un emploi sans et tiré du Bon Usage de Grevisse (édition 2008):

53) Je n'usais de mes médailles que pour rester en poste dans cette ville. Ce, pour la petite histoire, qui n'a apparemment rien à voir avec le fait et le sentiment de ce jour. (Y. Navarre, Portrait de Julien devant la fenêtre, cité par Hobæk-Haff, 2017: 94)

Elle montre aussi que le segment non verbal peut se trouver très en avant dans l'énoncé, pouvant s'insérer dans le contenu propositionnel:

54) Ensuite, et ce jusqu'à la fin de 1997, les équipes d'inspection seront composées pour $60 \%$ de représentants de partis politiques. (Le Monde cité par Hobæk-Haff, 2017:96)

L'analyse de Hobæk-Haff consiste à voir dans la seconde partie une proposition réduite:

55) Anne trompe son mari, et ce depuis longtemps = et (Anne trompe son mari) depuis longtemps.

Son analyse voit en ce une "anaphore de surface» et ce qui suit est analysé comme un focus. De fait, la structure en termes d'information est bien celle d'un démonstratif topique qui reprend en le transformant un contenu asserté antérieur, et son complément logique est le focus ou le commentaire. L'analyse de la focalisation renvoie à Nølke (1994, 2006): le focus est une information mise en valeur, saillante. Nølke 
distingue un focus neutre, souvent contraint par la position (finale) et un focus dû à une focalisation «spécialisée». La segmentation, mais aussi le support topique $c e$ qui aplanit en quelque sorte la reproduction de l'énoncé précédent, conduisent à la mise en évidence d'un focus spécialisé dans ce segment particulier.

À partir de cette analyse, il me semble qu'il se passe les choses suivantes dans ce type de construction:

- L'énoncé initial est analysable en topique et focus neutre s'il n'est pas segmenté. La segmentation, et elle seule, permet déjà un focus supplémentaire, attaché à un constituant qui étant disloqué, échappe en partie à l'organisation de l'énoncé verbal. S'il y a coordination, il se crée un effet d'ellipse du contenu verbal:

56) [...] il lui ordonna de ranger ces documents dans le tiroir aux oubliettes, et sans délai. (M. Lévy, cité par Hobæk-Haff, 2017: 93)

qui à lui seul va renforcer la focalisation sur le segment coordonné. Autrement dit, cela ne suffit pas pour expliquer ce qui se passe ici avec l'insertion de $c e$.

- L'occurrence de ce n'est possible qu'avec des constituants relativement autonomes et focalisables. Cela exclut un adverbe comme heureusement, selon Hobæk-Haff:

57) ${ }^{\star P}$ Pierre a tenu parole, et ce heureusement. (ibid. jugement de Hobæk-Haff, 2017: 93)

On peut aussi estimer que beaucoup d'adverbes, notamment ceux du premier cercle des ajouts, ne peuvent apparaître ${ }^{3}$ :

58) Il a bu, et beaucoup! / *Il a bu, et ce, beaucoup!

Il resterait à savoir quelles sont exactement ces limites d'emploi. La grande majorité des exemples attestés sont des locutions temporelles, il $\mathrm{y}$ a aussi des circonstanciels de lieu, et plus rarement des circonstanciels périphériques, comme le concessif dans:

3 Berrendonner (1990: 35) donne cet exemple avec ceci: C'est donc pour toutes ces raisons que je désire vivement ne plus faire partie de vos abonnés, et ceci au plus vite. L'adverbe est renforcé par "au plus», et le démonstratif a aussi la forme renforcée. Il me semblerait difficile de dire?? [...] et ce vite. Il semble aussi que ce soit d'usage plus contraint que ceci, cela. 
L'EMPLOI DE CE DANS LES REPRISES DE CONTENU PROPOSITIONNEL

59) D'ailleurs, votre vie durant, tous les actes publics seront établis à votre nom patronymique, et ce quel que soit votre état civil. (F-Magazine, juillet 1978, cité par Hobaek-Haff, 2017: 93)

Une condition nécessaire est que le constituant non verbal soit suffisamment détachable du noyau verbal intégré dans le contenu anaphorique de $c e$.

- Lorsque ce est possible, son utilisation ne me semble pas «superfétatoire» comme l'a écrit Berrendonner (1990: 35). Il réalise la transformation d'une structure prédicative complexe en un élément unique (qui devient en quelque sorte sa dénomination), sorte de topique minimal, accentuant par conséquent le contraste du contenu de l'information. Ce faisant, il fait du segment averbal bien autre chose qu'un circonstant détaché et focalisé: cet élément devient une assertion satellite complète, associant un topique à un focus, formant ainsi une prédication complète non autonome qui prend une importance et un relief que n'aurait jamais un circonstant détaché. Cela dit, il est généralement omissible, même en (59) ci-dessus, contrairement à ce que dit Hobaek-Haff. Il n'est obligatoire que lorsqu'il se trouve au début d'une phrase syntaxique comme en (53). Dans d'autres constructions son absence est gênante pour la délimitation de la partie anaphorisée:

7) Neuf heures, c'était déjà tard, même si on n'avait pas à se lever le lendemain pour traire les vaches, et ce pendant trois jours [...]. (Lafon)

L'absence de ce rend incertaine l'affectation du circonstanciel: il manque un relais qui fasse en quelque sorte le point sur ce qui va être le topique de ce prolongement, sans que cette absence soit agrammaticale.

\subsubsection{Sur ce, pour ce faire}

Avec sur, on a vu dans les exemples que la référence à ce qui précède est particulièrement vague. La construction en rappelle une autre également figée, sur ces entrefaites. Le clitique attaché à la préposition est ici un démonstratif qui renvoie à l'immédiat (ou presque) contexte antérieur, mais sans limites précises, pour fixer le début d'une nouvelle prédication. Contrairement à la construction examinée plus haut (2.1.1.), ce qui suit est indépendant: sur ce a le fonctionnement d'un connecteur, par succession dans le temps, de deux prédications. Les 
deux éléments de sur ce forment bloc. La construction est un vestige de l'époque où ce pouvait être en position finale accentuée (par exemple dans l'étymologie fantaisiste proposée par Rabelais du nom de la Beauce: Je trouve beau ce, dans Gargantua). Gougenheim (1974: 78) rappelle que la langue du $\mathrm{XVI}^{\mathrm{e}}$ siècle employait ce après les prépositions les plus diverses.

Le même clitique, antéposé comme en ancien français dans pour ce faire, renvoie, lui, à un contenu propositionnel plus circonscrit, qui décrit une action projetée. Le verbe ne doit pas faire illusion, ce n'est pas un verbe lexical indépendant mais la proforme verbale de sens englobant, forme archétype de l'action, représentée en français par faire. Il y a à mon sens une double anaphore, ou peut-être plus exactement une extension de l'anaphore au domaine du verbe, "faire cela» représentant une action (transitive ou non, ce n'est pas la question).

\subsubsection{La construction ce + participe présent}

Cette construction ce+ participe présent a des propriétés assez proches de pour ce faire: un ce proclitique, fixé dans une position d'objet préverbal remontant à l'ancien français, et l'emploi de proformes verbales, faire ou dire, selon le type d'action qui est anaphorisé. La position de ce est peut-être maintenue par la fonction discursive de connexion avec ce qui précède; mais, comme avec la construction infinitive, le segment est fonctionnellement rattaché à ce qui suit:

37) Alors on le voit tendre la main, dénouer un ruban entortillé et, ce faisant, converser avec l'enfant d'un air très naturel. (Garat)

Dans (37), la coordination relie le contenu propositionnel qui précède à la proposition infinitive qui suit. Le segment ce faisant s'y rattache comme une sorte de gérondif ( en faisant cela»). Dans tous les cas, un troisième segment est donc obligatoire. Le segment ce faisant devient alors plus ou moins un connecteur introduisant cette clause (cf. (39), où la séparation est nette).

\subsection{Les constructions à mot $Q u$ -}

\subsubsection{Les relatives pseudo-périphrastiques avec ce}

Cette construction est décrite par Sandfeld (1965, \$137, 138). Il souligne le rapport «peu étroit» de l'antécédent à la relative, et y voit 
L'EMPLOI DE CE DANS LES REPRISES DE CONTENU PROPOSITIONNEL

malgré la subordination une construction qui «équivaut à peu près», pour ce qui, à et cela. Les formes $Q u$ - de reprise de contenu prédicatif, aujourd'hui surtout observée avec l'association à une préposition (après quoi, sans quoi, grâce à quoi...) étaient aussi observables sans préposition en français classique, en particulier le qui sujet inanimé, devenu ce qui par la suite, lorsque le relatif précède le verbe être (Haase, $1965 \$ 35)$ :

60) Ce sera après que vous aurez gagné le procès de mon ami; qui sera cause, s'il vous plaît, que j’ajouterai un superlatif au bas de mes lettres. (Guez de Balzac, cité par Haase, 1965)

Chez Riegel et al. (2009: 815), qui propose cette dénomination de relative "périphrastique», il s'agit d'une sous-catégorie de toutes les relatives à tête démonstrative. Il me semble qu'elle n'est pas assez distinguée par sa principale caractéristique, qui est de désigner un contenu propositionnel non matérialisé précisément. Je rejoins sur ce point Kuyumcuyan (2011), qui parle de «pseudo-périphrastiques». La distinction est aussi faite par Hadermann (2014: 96) qui parle de «configuration discursive», caractérisée par l'absence de source lexicale pour former un antécédent dans la source de la relation anaphorique, et par le caractère non "unilatéral» de la relation entre cette source et le site du marqueur de subordination. Je comprends ce trait comme l'absence de toute fonction dans la "principale» en lien avec la subordonnée, d'où parfois l'absence même de relation de subordination dans certaines relatives circonstancielles. Cela dit, cette analyse ne distingue pas les formes avec ce, qui selon moi se caractérisent par une cohésion forte de type subordonné. Hadermann a cependant remarqué le rôle cohésif de $c e$ : «la présence du morphème ce explicite en quelque sorte un pointage vers une source propositionnelle antérieure» (ibid.: 96). L'analyse de Kuyumcuyan (2011) s'intéresse surtout à la référence, dont elle montre la variabilité et surtout la dépendance par rapport au contenu de la subordonnée qui doit également être pris en compte. Un de ses exemples le montre bien:

61) Partir à la retraite à 56 ans après avoir cotisé 43 ans signifie que l'on a quitté l'école à l'âge de 13 ans. Ce qui est strictement impossible: depuis le Front Populaire, la scolarité est obligatoire jusqu'à 14 ans, et jusqu'à 16 ans depuis 1959. (CE, 20/08/2008, dans Kuyumcuyan, 2011: 194) 
L'information dans la relative exclut comme sujet l'information "partir à la retraite» et doit se borner à la complétive. Elle décrit bien la topicalisation qui fait de ce le socle d'une nouvelle prédication active, malgré la subordination, à la manière des relatives "continuatives" (expression courante pour les analogues de ces énoncés en grammaire latine, allemande ou anglaise). Cette subordination réalise en effet une articulation souple avec ce qui précède. La rupture induite par la subordination permet, comme elle le constate, de réaliser «un énoncé sur un énoncé», un effet de récapitulation sur mesure puisque l'empan de ce qui est sélectionné par ce dépend du contenu de la subordonnée; la pseudo-périphrastique permet ainsi de changer de perspective, de passer du point de vue du personnage à celui du narrateur, de modifier les effets de saillance, d'orienter le discours vers un autre point de vue.

La relative peut aussi être analysée en termes de contenus sémantiques, on y verra des verbes «psychologiques», des «commentaires» au sens sémantique sur la source anaphorique, mais aussi, assez souvent, une suite de récit, une continuation. Le premier point à considérer, c'est la nature du lien associant ces relatives à leur antécédent. Voici un exemple de relative continuative classique (ce n'est pas une déterminative), tiré d'un article de Maurel:

62) Il ouvrit la porte, qu'il referma aussitôt. (Maurel, 1992: 78)

La relation à l'antécédent, qui ne peut être que le mot porte, ne peut se faire telle quelle: la porte dans la principale est fermée, si c'était celle-là, elle ne pourrait être refermée dans la subordonnée. Il se passe dans cette construction le même travail de réactualisation de la source de l'anaphore que dans la construction étudiée par Berrendonner à propos de ce: la référence de la porte dans la relative ne se fait qu'après que le locuteur a intégré le contenu cognitif dû à l'assertion en principale. La relative poursuit le récit entamé dans la principale et en tire les conséquences sur l'état du référent.

Maurel supposait ici ce qu'il nommait une «anadiplose»- la réduplication du nom seul, sous-jacent:

62a) Il ouvrit la porte, (porte) qu'il referma aussitôt.

Cette analyse syntaxique permet en effet de sauver une analyse de relative à antécédent, celui-ci étant enrichi par la prédication de la principale. Cela permet de décrire cette relative comme une relative adnominale, à fonction appositive, mais dotée de force énonciative. 
L'EMPLOI DE CE DANS LES REPRISES DE CONTENU PROPOSITIONNEL

On peut imaginer quelque chose d'analogue pour les relatives pseudo-périphrastiques: dans les constructions avec $c e$, on a affaire à une structure à antécédent, la mécanique de subordination est celle de ces relatives, avec les formes de complémenteur qui/que/dont qui ne sont pas autonomes, ont une morphosyntaxe basée sur les fonctions et remplacent des formes fortes, les véritables pronoms relatifs ${ }^{4}$ (pour cela, je renvoie à Muller 1996). De plus, tout ce qu'on a vu montre comment l'antécédent est une forme modifiée de sa source référentielle, qui est prédicative (des sous-structures de type prédicat verbal et arguments). La modification consiste à synthétiser sous la forme pronominale ce un contenu propositionnel. Cette forme nominalisée abolit de plus toute distinction entre prédicat et arguments, entre topique et commentaire dans la phrase-source, pour en faire un nouveau topique pour la suite, ce qui participe du dynamisme communicatif. Ainsi, ce ne représente pas exactement le contenu prédicatif, mais le produit de sa transformation en topique unifié, de la même façon que le mot sousjacent porte de (62a) n'est pas le mot analogue de la source référentielle. Étant topique, il exige une suite discursive qui va constituer la relative continuative.

Il me semble aussi que le terme n'est pas véritablement un démonstratif (il ne commute pas ici avec ceci/cela). Les raisons sont peut-être à chercher du côté de la morphosyntaxe, notamment de l'association étroite entre ce et le mot $Q u$ - qui suit. Quoi qu'il en soit, on a affaire à un pronom de première dénomination d'un référent non nommé, présenté comme second, en appendice, ce qui correspond assez bien à l'emploi de chose sans déterminant, alors que le ce indépendant vu ailleurs est toujours glosable par «cela». On trouve des relatives en $\operatorname{chose}^{5}$ qui reprennent du contenu propositionnel:

63) Mais la veuve avait de l'argent, chose qui invite les nègres comme Qualidor aux joies du mariage. (Chamoiseau, Texaco, 1992)

64) Mais aussi cette manie que j'avais de me mettre dans un état d'épuisement, d'atonie musculaire, en prenant très longtemps des bains trop chauds - chose qui m'était souvent, et avec raison, reprochée chez moi. (Duperey, Je vous écris, 1993)

4 Le pronom sous-jacent est uniformément le quoi «non humain » qu'on retrouve dans les reprises de contenu propositionnel lorsqu'il y a préposition $\left(\mathrm{P}_{0}\right.$, après quoi $\left.\mathrm{P}_{1}\right)$.

5 Cela a été remarqué par Sandfeld (1965, \$137: 224). 
65) J'entrevoyais tous les actes dont j'aurais pu me rendre capable (...) comme par exemple, au lieu de simplement chercher le nom de cette femme sur l'Internet, décharger sur elle un revolver en hurlant: "Salope! Salope! Salope!». Chose que je faisais d'ailleurs parfois, tout haut, sans revolver. (Ernaux, L'occupation, 2002)

Kleiber (1987) a rapproché le démonstratif de l'emploi de chose en reprise de contenu propositionnel, dans les constructions non subordonnées :

66) Paul gifla Berthe. Cela/La chose ne plut guère à la jeune fille (Kleiber, 1987: 116)

Il voit une analogie fonctionnelle entre les deux termes: il s'agit de désignateurs de «référents non nommés ou appréhendés comme tels». Il rappelle aussi que chose a failli devenir un outil démonstratif en français :

67) Puis avint chose li rois se combatié. (Charroi de Nîmes, 348)

(Puis il advint ceci, que le roi se mit à combattre)

Ce sens partagé de chose et de ce explique pourquoi il n'y a jamais d'antécédent nominal à ce type de relative. Il faudrait pour cela que le démonstratif ce puisse anaphoriser un nom, ce qui est exclu dans ce cas:

68) ${ }^{\star} I l$ ouvrit la porte, ce qu'il referma aussitôt.

Même avec le mot chose dans la première proposition, il n'y a pas de coréférence possible au nom dans la relative avec ce:

69) Il m'a rapporté de son voyage une chose, ce qui m'a surpris/

...chose qui m'a surpris.

Avec chose, deux sens bien distincts sont possibles: dans l'un, ce terme est la reprise par répétition immédiate ou anadiplose, du terme nominal; dans l'autre, chose nominalise l'ensemble de la prédication antérieure. Il apparaît donc que le ce s'est spécialisé dans cette dernière valeur.

Il est intéressant pour l'analyse du démonstratif dans ce contexte de voir que son correspondant nominal chose se présente sans article devant la relative. Il y a quelque chose de commun avec la reprise anaphorique sous forme d'anadiplose décrite par Maurel, même si le terme est peut-être impropre formellement puisqu'il n'y a pas de premier terme que ce ou chose peuvent reprendre. Il se peut qu'on ait 
le même fonctionnement: le contenu propositionnel est mentalement un "cela», une "chose» dont l'occurrence réalisée est présentée en quelque sorte comme seconde, formant le socle à partir duquel la relative va se développer. Comme le dit Kuyumcuyan (2011: 202), «la saillance est peut-être dans la mémoire discursive plutôt que dans le texte». Je dirais que la nominalisation comme processus précède sa reprise par ce qui la matérialise. Cela donne une véritable spécificité à cette classe de relatives.

Il faut peut-être distinguer les constructions basées sur des conjonctions fonctionnelles, surtout qui, que, des relations basées sur des pronoms $\mathrm{Qu}$ - présents. Le ce est obligatoire avec qui, que, dont et sauf peut-être pour dont, un verbe conjugué doit suivre (sauf archaïsme comme dans ce que voyant). Il y a donc une structure propositionnelle complète.

Dans de nombreux cas, le ce est facultatif lorsqu'on a affaire à un relatif quoi, et dans d'autres, il est totalement exclu, comme le montre Lefeuvre (2006: 243). Il semble que le ce soit surtout présent avec des relatifs liés par des relations fonctionnelles étroites au verbe de la relative (ce à quoi, ce en quoi, ce sur quoi, ce contre quoi) et qu'il est absent avec des adverbes circonstanciels comme après quoi.

La facultativité est explicable par la présence du pronom plein; en somme pour à quoi, on peut avoir ce à quoi avec le sens «chose à laquelle» ou bien à quoi avec le sens «à laquelle chose». Dans le premier cas, on retrouve la structure informationnelle déjà vue, dans laquelle quoi tout en marquant la fonction est anaphore de ce pour la subordonnée, l'ensemble formant un ensemble topique/commentaire; dans le second cas, le lien ne se fait que dans le relatif, à cause de l'anaphore pronominale portée par le seul quoi. La différence est légère quand on a une fonction argumentale dans la subordonnée, simplement on n'a pas de distinction nette dans l'ensemble topique/commentaire. Par contre, lorsqu'il s'agit d'un circonstant, il en va autrement: l'adjonction de ce est bloquée, notamment lorsque la préposition introduisant quoi n'a pas de fonction réelle dans la subordonnée. On a parfois de fausses relatives, ou plutôt une relative compactée suivie d'une indépendante:

70) Ils y mirent le feu impitoyablement, sans être touchés des cris de cette malheureuse. Après quoi, ils coururent les rues toute la nuit, brisèrent un nombre infini de lanternes. (Chandernagor, L'allée $d u$ roi, cité par Lefeuvre 2006: 236) 
Lefeuvre fait remarquer qu'on ne peut dire:

70a) ${ }^{\star}$ Ce après quoi ils coururent les rues toute la nuit [...]. (ibid.: 243)

On peut noter que cette relative serait parfaitement possible si le verbe subordonné signifiait "courir après quelque chose». Il me semble qu'ici, et c'est pourquoi on peut supposer qu'on est passé à une interprétation de connecteur, le verbe subordonné n'est pas véritablement en relation grammaticale avec après quoi. La virgule va dans le sens d'une interprétation de clausule relative complète "après laquelle chose (étant faite)", ce qui est rendu possible parce qu'il n'y a pas ici de conjonction. On pourrait donc avancer, ce qui devrait être vérifié par des recherches plus approfondies, que ce devant un pronom $Q u$ - pourrait être utilisé pour faire le lien entre le référent phrastique et le contenu argumental induit par la subordonnée.

\subsubsection{La construction ce que + participe présent}

Il ne subsiste guère de cette construction autrefois usuelle ${ }^{6}$ que ce que voyant, même s'il existe quelques attestations de ce que faisant. Il n'y a pas tellement de différence perceptible entre les deux constructions ${ }^{7}$, avec ou sans que, même si on observe une répartition des rôles: sans que, avec les proformes verbales d'action et de parole; avec que, pour le verbe voyant. On a formellement une relative, et $c e$ que est la version moderne du quoi qui a disparu de la rection directe. La relative participiale est également subordonnée à la clause qui la suit, tout en fonctionnant comme connecteur avec celle qui précède.

\subsubsection{Les structures: $\mathrm{P}, c^{\prime} e s t$ ce + relative}

La structure fait de l'ensemble topique+commentaire vu précédemment un terme focalisé, la relation est facile à établir puisque ce reprend comme attribut le contenu de $\mathrm{P}$, nominalisé une première

6 Gougenheim en donne des exemples dans la langue du $\mathrm{xvI}^{\mathrm{e}}$ siècle: Ce que considérant, [...]. (Montaigne); Ce que craignans les juges feirent mettre des gens armez alentour d'eulx (Amyot) (Gougenheim, 1974: 93, 97)

7 Leur origine est distincte: Ce faisant/ce disant sont des participiales, pas des relatives, avec un clitique objet antéposé, position encore usuelle au XvI ${ }^{\mathrm{e}}$ siècle. Par exemple, avec voyant: Ce voyant, Pantagruel en voulut autant faire. (Gougenheim, 1974: 78). 
fois ${ }^{8}$ comme sujet du verbe être de la clivée. Il y a donc une relation équative qui est ici rendue explicite. Il me semble que le résultat de cette focalisation est aussi de donner plus d'autonomie au contenu de la relative: la fonction n'est plus celle d'une adjonction apposée, mais d'une véritable phrase (il s'agit toujours de phrases distinctes dans nos exemples) qui est l'aboutissement focalisé d'une première assertion. Il est alors possible d'avoir des énonciateurs distincts, comme dans:

36) -Que diriez-vous d'aller voir où en sont les travaux à l'église Notre-Dame? - Comment! s'écria le duc, ils ne sont pas encore terminés? - C'est ce dont nous nous rendrons compte. (Queneau)

Le ce réfère ici au contenu propositionnel de l'énoncé interrogatif qui précède.

\section{Conclusion}

On a exploré le domaine des reprises de contenu propositionnel utilisant ce. À côté des constructions plus ou moins figées, telles que sur ce, ou les formes à complément antéposé, se présentent plusieurs constructions qui ont une originalité certaine, peut-être pas assez mise en évidence dans les études sémantiques et syntaxiques des liaisons de propositions. C'est d'une part, la construction en appendice averbal à ce topique, qui demanderait des examens plus approfondis, notamment pour le rôle du démonstratif, en comparaison avec les autres formes de démonstratif, et aussi les relatives très particulières qui permettent comme on l'a vu, à partir du ce qui à la fois nominalise et reprend un contenu propositionnel, d'enclencher une dynamique de continuation dans laquelle le contenu propositionnel recyclé en nominal forme le socle topique à de nouveaux développements.

\section{Bibliographie}

BERRENDONNER A. (1990), Pour une macrosyntaxe, Travaux de linguistique 21, 25-36.

$8 \quad$ Il n'y a pas le lien étroit entre segments qu'illustrent les structures pseudo-clivées étudiées par Roubaud (2000): le contenu propositionnel initial n'est ici jamais focalisé avant l'apparition de c'est que. 
BURIDANT C. (2000), Grammaire nouvelle de l'ancien français, Paris, SEDES.

GOUGENHEIM G. (1974), Grammaire de la langue française du $16^{e}$ siècle, Paris, Picard.

GUILLOT C. (2006), Démonstratif et deixis discursive: analyse comparée d'un corpus écrit de français médiéval et d'un corpus oral de français contemporain, Langue française 152, 56-69.

GREVISSE M. (198011), Le bon usage, Gembloux, Duculot.

HAASE A. (1965 $\left.{ }^{5}\right)$, Syntaxe française du XVII siècle, Paris, Delagrave.

HADERMANN P. (2014), La construction corrélative et les marqueurs en $\mathrm{Qu}$-, Langue française 182, 91-106.

HOBÆK-HAFF M. (2017), La construction en et ce revisitée, et ce par Henning Nølke, in Birkelund M., (éd), Points de vue, Mélanges offerts à Henning Nølke à l'occasion de sa retraite, Aarhus, Université d'Aarhus, 90-101.

KLEIBER G. (1987), Mais à quoi sert donc le mot chose?, Langue française 73, 109-128.

KUYUMCUYAN A. (2011), Syntaxe et sémantique du relatif de liaison en français moderne, Annales littéraires de l'Université de Franche-Comté 897, 187-204.

LEFEUVRE F. (2006), Quoi de neuf sur quoi?, Rennes, Presses universitaires de Rennes.

MAUREL J.-P. (1992), Subordination inverse et neutralisation du relatif, Travaux linguistiques du Cerlico 5, 72-98.

MULLER C. (1996), La subordination en français, Paris, A. Colin.

NØLKE, H. (1994), Linguistique modulaire: de la forme au sens, Louvain-Paris, Peeters.

NØLKE, H. (2006), La focalisation: une approche énonciative, in Wlodarczyk H. et A. (éds), La focalisation dans les langues, Paris, L'Harmattan, 109-119.

RIEGEL M., PELLAT J.-C., RIOUL, R. (20094), Grammaire méthodique $d u$ français, Paris, Presses universitaires de France.

ROUBAUD M.-N. (2000), Les constructions pseudo-clivées en français contemporain, Paris, Champion.

SANDFELD K. $\left(1965^{2}\right)$, Syntaxe du français contemporain, Les propositions subordonnées, Genève, Droz. 